\title{
La estimulación cognitiva. Base para el proceso de la lectoescritura en estudiantes con discapacidad intelectual.
}

DOI: https://doi.org/10.33262/ap.v3i3.2.100

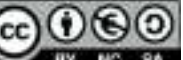

Cognitive stimulation. Basis for the process of literacy in students with intellectual disabilities.

Janeth Margarita Quimi Quimi. ${ }^{1}$ \& Giceya de la Caridad Maqueira Caraballo. ${ }^{2}$

\begin{abstract}
Introduction. Cognitive stimulation from an early age constitutes a fundamental premise for the training of learning processes in children, especially in those with an intellectual disability. Objective. To analyze the influence of cognitive stimulation in the process of acquisition of literacy in students with intellectual disabilities in elementary basic education. Methodology. The investigative procedure was determined by a type of descriptive research, supported by theoretical and empirical methods. For the collection of information, different instruments were used within these, an observation guide, a questionnaire and an estimation scale, which favored the obtaining of data of great interest. Results. 1. The theoretical referents consulted corroborate the importance and usefulness of cognitive stimulation for the literacy learning process in students with intellectual disabilities. 2 . The existing limitations in teachers caused by ignorance about how to perform cognitive stimulation for the process of acquisition of literacy in students with intellectual disabilities are determined. 3. It is necessary to design a didactic strategy that, considering its structure and components, allows it to positively influence the development of cognitive stimulation for the learning of literacy in students with
\end{abstract}

\footnotetext{
${ }^{1}$ Universidad Laica Vicente Rocafuerte. yquimiq@ulvr.edu.ec. https://orcid.org/0000-0002-7382-5981

${ }^{2}$ Universidad Laica Vicente Rocafuerte. gmaqueirac@ ulvr.edu.ec. https://orcid.org/ 0000-0001-62823027
} 
disabilities. Conclusions. The importance of cognitive stimulation in students with disabilities is reaffirmed, with special interest in learning to read and write. A didactic strategy was designed, which considering its structure and components was favorably validated by the specialists consulted, confirming its usefulness in the process of cognitive stimulation for the learning of literacy in students with intellectual disabilities. The application of the proposed proposal could positively influence the solution of the existing problem, notably contributing to improving the literacy learning process in these students.

Keywords: cognitive stimulation, learning, intellectual disability, literacy.

\section{Resumen}

Introducción. La estimulación cognitiva desde edades tempranas constituye una premisa fundamental para el entrenamiento de los procesos de aprendizaje en los niños, especialmente en aquellos que presentan una discapacidad intelectual. Objetivo. Analizar la influencia de la estimulación cognitiva en el proceso de adquisición de la lectoescritura en estudiantes con discapacidad intelectual de la enseñanza básica elemental. Metodología. El proceder investigativo estuvo determinado por un tipo de investigación descriptiva, apoyada en métodos teóricos y empíricos. Para la recolección de información se emplearon diferentes instrumentos dentro de estos, una guía de observación, un cuestionario y una escala de estimación, los cuales favorecieron la obtención de datos de gran interés. Resultados.1. Los referentes teóricos consultados corroboran la importancia y utilidad de la estimulación cognitiva para el proceso de aprendizaje de la lectoescritura en los estudiantes con discapacidad intelectual. 2. Se determinan las limitaciones existentes en las docentes provocadas por el desconocimiento sobre cómo realizar la estimulación cognitiva para el proceso de adquisición de la lectoescritura en los estudiantes con discapacidad intelectual. 3. Se requiere diseñar una estrategia didáctica, que considerando su estructura y componentes permita influir positivamente en el desarrollo de la estimulación cognitiva para el aprendizaje de la lectoescritura de los estudiantes con discapacidad. Conclusiones. Se reafirma la importancia de la estimulación cognitiva en los estudiantes con discapacidad, con especial interés en el aprendizaje de la lectoescritura. Quedo diseñada una estrategia didáctica, que considerando su estructura y componentes fue validada favorablemente por los especialistas consultados, confirmando su utilidad en el proceso de estimulación cognitiva para el aprendizaje de la lectoescritura de los estudiantes con discapacidad intelectual. Según valoración realizada la aplicación de la propuesta planteada pudiese incidir de manera positiva en la solución de la problemática existente, contribuyendo notablemente a mejorar el proceso de aprendizaje de la lectoescritura en estos estudiantes.

Palabras claves: estimulación cognitiva, aprendizaje, discapacidad intelectual, lectoescritura. 


\section{Introducción}

La atención temprana a los menores que presentan cualquier tipo de necesidad educativa especial, asociada o no a una discapacidad sigue constituyendo una prioridad educativa y social. En el caso de los que tienen una discapacidad intelectual lo anterior es de extrema importancia, precisamente porque dependiendo del nivel de afectación que presenten y de la estimulación que reciben en los primeros años de vida, así serán las posibilidades de inclusión educativa y social que se logre, es por ello que estimular el desarrollo cognitivo desde edades tempranas en los menores con discapacidad intelectual ha sido tema de análisis por diversos autores, dado su incidencia en el posterior desarrollo integral del niño.

Según Arango, Infante \& López, (2006), la estimulación temprana son acciones dirigidas a ofrecerle a los niños las experiencias que necesitan, desde el propio nacimiento, para que puedan desarrollar al máximo nivel su potencial biopsicosocial.

En correspondencia con lo señalado, es importante considerar los aportes de Albornoz, \& Guzmán, (2016), al referirse a la importancia de la estimulación temprana, explicando que es necesario proporcionar una estimulación adecuada en el momento oportuno y ello debe realizarse desde las etapas tempranas de la vida de los niños.

Al respecto Paiget, (1973) conceptualizo el primer periodo, de 0 a 24 meses, como de inteligencia sensorio-motriz, señalando que se refiere a la capacidad de dar solución a los problemas que enfrenta, partiendo de las actividades de percepción, las actitudinales, de tono, así como a los movimientos sin referencias a evocaciones simbólicas, o sea antes de que aparezca el lenguaje expresivo verbal. Esa inteligencia, según Paolini, Oiberman, Mansilla, (2017), se desarrolla en un plano sensorio-motor, mediante las acciones y de los sentidos, correspondiendo a una inteligencia practica enmarcada antes del surgimiento del lenguaje expresivo.

Pollitt, \& Caycho, (2012), son del criterio que de manera general en la etapa menor a tres años, el desarrollo psicomotor es evaluado como un indicador del desarrollo integral del niño, no obstante Paolini, Oiberman, Mansilla, (2017), plantean que en innumerables ocasiones el área de la cognición no se corresponde a las etapas de desarrollo esperadas, evidenciándose un desfasaje entre el desarrollo cognitivo y el resto de las áreas, tales como la de lenguaje, motricidad-coordinación y la propia conducta social.

Es por ello que Morales, (2017), indica que la estimulación cognitiva tiene como premisa el entrenamiento de las capacidades cognitivas en igual medida que las motoras, explicando que si se ejercitan de forma sistemática tienden a responden de favorablemente y por ende repercuten en el desarrollo integral del niño. En esta dirección se coincide con los fundamentos teóricos precedentes en cuanto a la importancia y utilidad de la estimulación cognitiva, precisamente por su incidencia y relación con el aprendizaje de la lectoescritura. Se comprende que mientras mayor sea la estimulación recibida, mejor desempeño en el aprendizaje se puede lograr, entre otros aspectos porque la 
estimulación cognitiva unida a la estimulación motriz constituye la base para el desarrollo posterior del niño, incidiendo favorablemente en el aprendizaje de la lectoescritura.

En este contexto, Azcoaga, (1986) y Rivera, (2016), plantean que, a nivel cognitivo, es fundamental recalcar que los niños realizan su aprendizaje a través de la experiencia, por lo que, su cerebro necesita registrar y evidenciar tanto los estímulos como la información adecuada, para poder responder acertadamente. Según indica el autor de referencia esta capacidad da lugar a que se comience a estimular diferentes procesos, dentro de estos es muy importante mediante diferentes actividades y estímulos estimular la atención, lo cual es muy beneficioso para el niño, porque de esta forma su cerebro va creando nuevas acciones y edificando su propio conocimiento de manera significativa.

Siguiendo este orden de ideas es oportuno considerar los aportes de García, (2013), al indicar que: el proceso atencional comienza a aparecer a partir de los 2 y 3, desarrollándose de manera gradual, pasando por la etapa escolar donde debido a la responsabilidad académica se da una atención sostenida, hasta llegar a los 8 y 10 años en que se inicia el desarrollo de la atención selectiva.

Según la American Association on Intellectual and Developmental Disabilities (AAIDD) (2011), el termino discapacidad intelectual se emplea para denominar a aquellas personas que presentan capacidades cognitivas y habilidades adaptativas disminuidas tanto en intensidad y en sus distintos grados manifestándose desde los primeros años de sus vidas., sobre todo al compararlas con la gran mayoría de la población,

En este ámbito, diversos autores, dentro de los cuales se cuenta Flores, (2018), afirman que la discapacidad intelectual es un estadio del individuo caracterizado por limitaciones significativas ya sea en el funcionamiento intelectual como en la conducta adaptativa, las cuales se refieren a las habilidades adaptativas conceptuales, sociales y prácticas, que se originan antes de los 18 años.

Ahora bien, atendiendo a lo abordado es meritorio destacar que la estimulación cognitiva resulta imprescindible cuando estamos ante niños que presentan discapacidad intelectual, dado que debido a sus propias características necesitan de varios niveles de apoyos y adaptaciones. Lo cierto es que cuando se trabaja la estimulación cognitiva de manera progresiva mediante las actividades pedagógicas, apoyos y niveles de ayudas ofrecidas, potenciamos su propia autonomía y su relación con el entorno, ayudando a que estos sean más independientes y puedan incluirse favorablemente tanto al contexto educativo como social.

En atención con los aportes y presupuestos teóricos precedentes, el aprendizaje de la lectoescritura ocupa un lugar prioritario en los estudiantes con discapacidad intelectual. La lectura como se conoce es fuente inagotable de innumerables aprendizajes y experiencias, mediante su práctica se activan las funciones cognitivas, se agudiza la inteligencia, incidiendo favorablemente en el desarrollo de la creatividad y fantasía de los menores. Otra de las bondades que se identifican con la lectura es que precisamente su aprendizaje promueve el esfuerzo, conduciendo a la estimulación de la atención y 
concentración del niño, lo que se traduce en un mejor rendimiento académico. En relación a la escritura, la misma contribuye a la organización y estructuración del pensamiento, pudiendo incidir incluso en los propios sentimientos de los niños, lo que origina alcanzar niveles de expresión más elaborados.

Como resultado de lo anterior, Bravo, (2016), explica que la enseñanza de la lectoescritura conlleva irreparablemente a incorporar al niño en la búsqueda de los significados de las letras, silabas y palabras del texto, o sea, constituye una senda para el desarrollo de su pensamiento. Seguidamente añade que, desde la óptica de los niños, el aprendizaje de la lectoescritura constituye la culminación del desarrollo cognitivo iniciado desde que nace y que evoluciona conforme a las condiciones que existan en su ambiente familiar y socio cultural.

Por supuesto que para la realización de la lectoescritura es fundamental dominar las capacidades preacadémicas y lingüísticas que se aprenden con anterioridad a la entrada de los niños a la escuela. En concordancia con Rugerio y Guevara (2015), estas capacidades incluyen elementos del desarrollo motor, tanto fino como grueso, comprensión verbal, pronunciación, distinción de sonidos, manejo de vocabularios básico, comprensión de narraciones y aptitudes de la comprensión numérica.

Se comparte con González (2020), al explicar que la lectoescritura es un proceso complejo, cuya misión es la enseñar a leer y a escribir y para su implementación se cuenta con elementos esenciales como la respiración, el vocabulario, la hidratación, el habla, el escuchar, visualizar, la psicomotricidad, así como otras habilidades del pensamiento como la observación y la identificación, la descripción, las habilidades motrices, y las emocionales y lingüísticas.

Sin embargo, independientemente al reconocimiento y valía que tiene para el desarrollo integral de los menores con discapacidad intelectual la estimulación cognitiva, con énfasis en la estimulación y aprendizaje de la lectoescritura, todavía persisten situaciones que impiden el manejo adecuado de este proceso en el contexto escolar, entre otros aspectos por las limitaciones existentes en el claustro docente para provocar el desarrollo de estrategias didácticas conducentes a la estimulación cognitiva de los estudiantes con discapacidad intelectual. En sintonía con lo señalado la investigación tiene como objetivo analizar la influencia de la estimulación cognitiva en el proceso de adquisición de la lectoescritura en estudiantes con discapacidad intelectual de la enseñanza básica elemental.

\section{Metodología}

En la investigación se fundamenta la incidencia que posee la estimulación cognitiva en la adquisición de la lectoescritura en niños con discapacidad intelectual, ubicados en la enseñanza básica elemental, ubicados en la Unidad Educativa Fiscal Iliana Espinel Cedeño de la ciudad de Guayaquil. 
El proceder investigativo estuvo sustentado en los aportes de Díaz et al. (2016), determinándose para su desarrollo un tipo de investigación descriptiva, la cual estuvo apoyada en métodos teóricos, dentro de estos: el histórico lógico, el analítico sintético, inductivo deductivo y el sistémico estructural y funcional, como parte de los métodos empíricos se empleó la observación. Para la recolección de información se utilizaron diferentes instrumentos tales como: guía de observación, el cuestionario y una escala de estimación, los cuales favorecieron la obtención de datos de gran interés.

La muestra estuvo estuvo integradas por 4 docentes que laboran en este nivel de enseñanza y 10 estudiantes que presentaban discapacidad intelectual, pertenecientes al nivel de básica elemental, para un total de 14 personas participantes. Todos pertenecientes a la Unidad Educativa Fiscal Iliana Espinel Cedeño de la ciudad de Guayaquil.

Dentro de los criterios de inclusión establecidos para la selección de la muestra de docentes se determinaron los siguientes:

- Laborar en la Unidad Educativa de referencia en el nivel básico elemental

- Estar dispuestos a participar de la investigación.

- Tener dentro del curso estudiantes con discapacidad intelectual

Los criterios de inclusión determinados para seleccionar los estudiantes participantes fueron:

- Presentar una discapacidad intelectual

- Estar cursando el nivel de enseñanza básica elemental

- Presentar dificultades en el aprendizaje de la lectoescritura.

\section{Resultados}

Con la aplicación de los instrumentos a la población objeto de estudio, se obtuvieron relevantes resultados, que permitieron conocer con exactitud la situación problemática existente.

La investigación constató que el $75 \%$ de la población docente encuestada, consideró que la estimulación cognitiva, presenta una importancia significativa dentro de los procesos de enseñanza aprendizaje; sin embargo, según el 50\%, la aplicación en el aula de las técnicas de estimulación cognitiva se realizan con muy con poca frecuencia, por lo que se hace muy necesario incrementar su aplicación, así como, aplicar otras alternativas y estrategias de estimulación (75\%), factibles a ser incluidas dentro de sus horas clases, que favorezcan al óptimo desarrollo de los estudiantes que presentan discapacidad intelectual.

De igual manera se determinó que las estrategias utilizadas para estimular la parte cognitiva de los niños, carecen de dinamismo y motivación, comprobándose que el 50\% de los docentes realiza la estimulación con ejercicios de memorización, constituyendo 
posiblemente una de las causas que evidencie el porqué los estudiantes no muestran avances tan significativos en los procesos de adquisición de la lectoescritura.

No obstante a conocer que los procesos de aprendizaje en esta población son más lentos, también es importante notar que de acuerdo a lo evidenciado en teorías e investigaciones previas, la estimulación cognitiva favorece sus procesos de avance; en este sentido, al no estar recibiendo esta población de estudiantes, estrategias adecuadas que contribuyan con este hecho, el $100 \%$ de los docentes afirman que la adquisición de las habilidades de lectura y escritura se torna más lenta aún de lo esperado, por lo que se precisa necesariamente, incidir en que se mejore la praxis docente.

En este sentido, es real que los docentes al elaborar sus estrategias de estimulación cognitiva pueden encontrar diferentes dificultades, no obstante, los resultados obtenidos después de aplicado los instrumentos, demuestran que en general la dificultad que prevalece $(75 \%)$, es el desconocimiento que poseen los docentes sobre las estrategias puntuales para la atención oportuna de los estudiantes con discapacidad intelectual, ocasionando por ende, que la educación impartida a esta población de estudiantes, actualmente no cumpla con todo lo establecido repercutiendo negativamente en todo lo relacionado con los principios de calidad, igualdad de condiciones y oportunidades para todos.

Por otra parte, en las encuestas aplicadas, dio como resultado que la generalidad de los docentes encuestados (75\%), reconocieron que no conocen suficientemente lo relacionado con las adaptaciones curriculares que les permita abordar de manera adecua el trabajo con la lectoescritura en los estudiantes con discapacidad intelectual, reflejándose este aspecto, en los niveles bajo de aprendizaje que presentan los mismos comparado con el nivel de aprendizaje esperado, en correspondencia a su condición y al nivel educativo que cursan, pudiéndose obtener mejores resultados de aplicarse las estrategias de adaptación curricular adecuadas.

También en la investigación, se demostró que los estudiantes objeto de la investigación, merecen recibir una mayor estimulación dado que a pesar de contar con habilidades primarias de lectura, el $75 \%$ de los docentes no han generado las alternativas adecuadas que permitan estimular el área cognitiva, pues se ha demostrado por investigaciones anteriores, que favoreciendo los procesos de concentración, atención, memoria, percepción y praxias entre otros, se contribuye de manera determinante a la adquisición del lenguaje escrito que conlleve a favorecer en la educación igualitaria a los que tienen derecho conforme a la normativa vigente.

Lo anterior, evidencia tres ideas fundamentales como resultado del proceso investigativo:

1. Los referentes teóricos consultados corroboran la importancia y utilidad de la estimulación cognitiva para el proceso de aprendizaje de la lectoescritura en los estudiantes con discapacidad intelectual. 
2. Se determinan las limitaciones existentes en las docentes provocadas por el desconocimiento sobre cómo realizar la estimulación cognitiva para el proceso de adquisición de la lectoescritura en los estudiantes con discapacidad intelectual.

3. Se requiere diseñar una estrategia didáctica, que considerando su estructura y componentes permita influir positivamente en el desarrollo de la estimulación cognitiva para el aprendizaje de la lectoescritura de los estudiantes con discapacidad.

\section{Propuesta de solución.}

\section{Estrategia Didáctica de Estimulación Cognitiva para el aprendizaje de la} lectoescritura en estudiantes con discapacidad intelectual.

Con la finalidad de dar solución a la problemática suscitada en la investigación realizada, se diseñó una estrategia didáctica para la estimulación cognitiva y aprendizaje de la lectoescritura en estudiantes con discapacidad intelectual. Es importante destacar que para la elaboración de la estrategia didáctica se tomó en cuenta la generalidad del contexto en el que debe ser aplicada, teniendo en cuenta los recursos necesarios para su implementación, la disposición de los docentes participantes y la factibilidad y viabilidad de la misma, la cual fue valorada positivamente por los especialistas consultados.

Figura No. 1. Flujo de la Propuesta de Estrategia para la Estimulación Cognitiva en estudiantes con Discapacidad Intelectual.

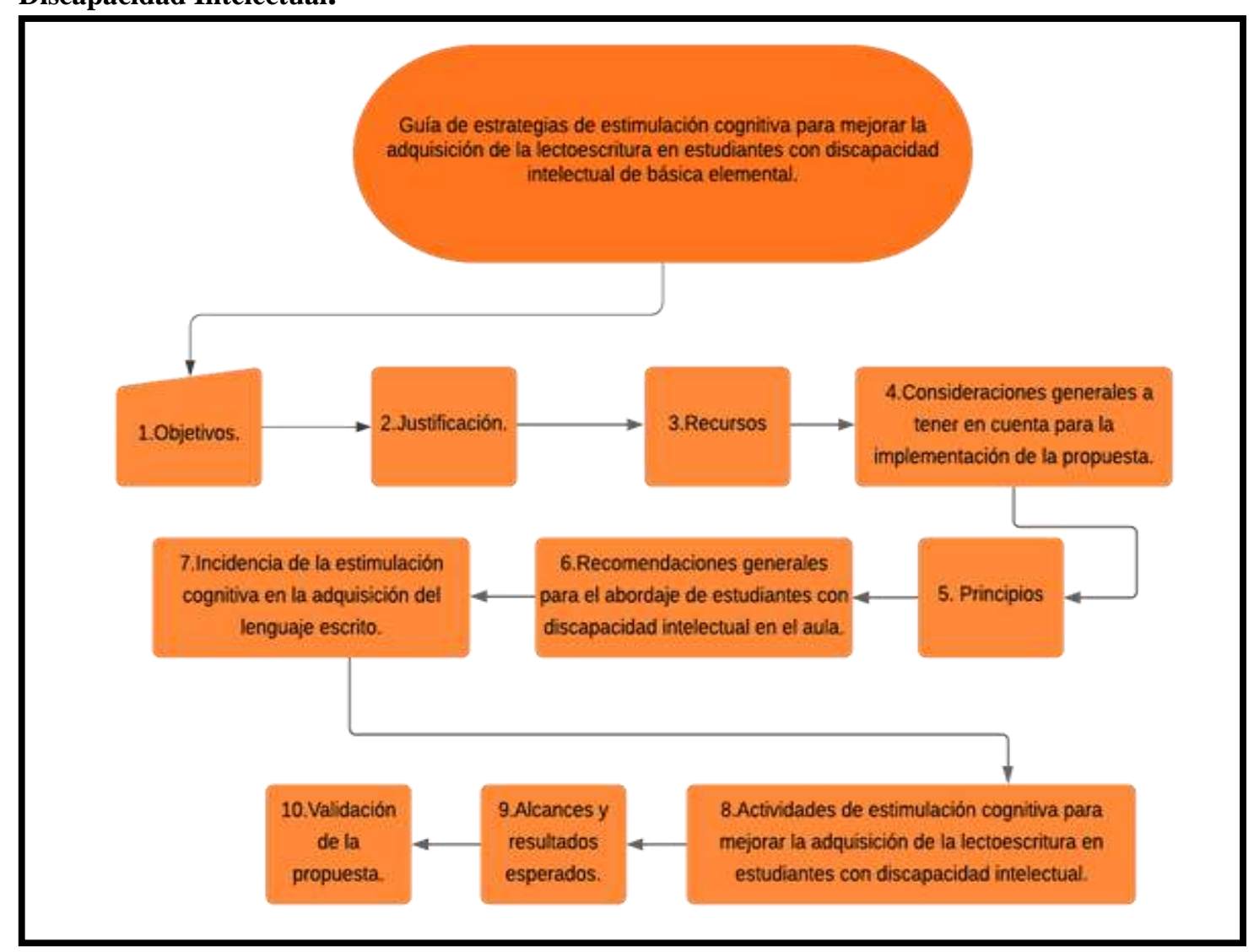

Elaborado por Quimi, 2021 


\section{Ejemplo de actividad a realizar.}

\section{Actividad No 1.}

Título: La rayuela de las palabras.

Objetivo: Lograr que el estudiante realice una asociación visual para el reconocimiento y reproducción de grafemas.

Inicio: Colocar alrededor de todas las instalaciones de la unidad educativa muchas letras en diferentes lugares que puedan ser visibles para los estudiantes.

Desarrollo: Se entrega al estudiante una letra y se le indica que busque la letra igual a la entregada entre todas las letras ubicadas a su alrededor, posteriormente el estudiante debe traer la letra encontrada.

Cierre y evaluación: El estudiante deberá contar las letras encontradas y escribir en el pizarrón la letra tantas veces como la haya encontrado.

Recursos: letras, cinta adhesiva, pizarrón, marcador, espacios diversos de la institución

\section{Conclusiones}

- Las conclusiones obtenidas de la investigación realizada se concretan en:

- Se reafirma la importancia de la estimulación cognitiva en los estudiantes con discapacidad, con especial interés en el aprendizaje de la lectoescritura.

- Quedo diseñada una estrategia didáctica, que considerando su estructura y componentes fue validada favorablemente por los especialistas consultados, confirmando su utilidad en el proceso de estimulación cognitiva para el aprendizaje de la lectoescritura de los estudiantes con discapacidad intelectual.

- Según valoración realizada la aplicación de la propuesta planteada pudiese incidir de manera positiva en la solución de la problemática existente, contribuyendo notablemente a mejorar el proceso de aprendizaje de la lectoescritura en estos estudiantes.

\section{Referencias Bibliográficas}

Albornoz, E, J., \& Guzmán, M., (2016). Desarrollo cognitivo mediante estimulación en niños de 3 años. Centro desarrollo infantil Nuevos Horizontes. Quito, Ecuador. Universidad y Sociedad [seriada en línea], 8 (4). pp. 186-192. Recuperado de http://rus.ucf.edu.cu/

Asociación Americana de Discapacidades Intelectuales y del Desarrollo (AAIDD). Discapacidad intelectual. Definición, clasificación y sistemas de apoyo. Alianza Editorial, Madrid 2011.Artículos científicos, tipos de investigación y 
productividad científica en las Ciencias de la Salud. Revista Ciencias de la Salud, vol. 14, núm. 1, 2016, pp. 115-121 Universidad del Rosario Bogotá, Colombia.

Arango de Narváez M, Infante de Ospina E., \& López de Bernal M., (2006). Estimulación Temprana. Bogotá: Ediciones Gamma. ISBN: 958-9308-14-7 volumen I; 9589308-15-5 volumen II; 958-9308-16-3 volumen III.

Azcoaga, J., (1986). Aprendizaje fisiológico. Psicología. Lenguaje. Aprendizaje (Actas de las Primeras Jornadas Nacionales de APINEP, Rosario, 1986). Buenos Aires, Ediciones Pedagógicas, 1987, 17-32.

Bravo, 1., (2016)- El aprendizaje del lenguaje escrito y las ciencias de la lectura. un límite entre la psicología cognitiva, las neurociencias y la educación. Límite. Revista Interdisciplinaria de Filosofía y Psicología Volumen 11, No 36, 2016, pp. 50-59. Pontificia Universidad Católica de Chile.

Díaz-Narváez, Víctor Patricio; Calzadilla Núñez, Aracelis (2016)- Artículos científicos, tipos de investigación y productividad científica en las Ciencias de la Salud Revista Ciencias de la Salud, vol. 14, núm. 1, 2016, pp. 115-121 Universidad del Rosario Bogotá, Colombia.

Flores, J., (2018)- La comprensión actual de la discapacidad intelectual. Sal Terrae | 106 (2018) 479-492

García, G., (2013)- Análisis neuropsicológico de la atención, memoria y funciones ejecutivas en niños con trastorno por déficit de atención con hiperactividad (TDAH). Universidad Autónoma de Nuevo León.

González, M., (2020)- Habilidades para desarrollar la lectoescritura en los niños de educación primaria. Revista Estudios en Educación.Vol.3, Nº, julio de 2020.ISSN en línea 2452-4980 • pp.45-68

Morales, E., (2017)- Estimulación cognitiva y la lecto-escritura de los estudiantes de tercer año de Educación General Básica de la Unidad Educativa Julio Enrique Fernández, parroquia Izamba del Cantón Ambato. Carrera de Psicología Educativa. Universidad Técnica de Ambato. Ecuador.

Paolini, C., Oiberman A, Mansilla M (2017)- Desarrollo cognitivo en la primera infancia: influencia de los factores de riesgo biológicos y ambientales- Universidad de Ciencias Empresariales y Sociales. https://www.redalyc.org > journal > html

Pollitt, E., \& Caycho, T., (2012). "Desarrollo motor como indicador del desarrollo infantil durante los primeros dos años de vida”. Revista de Psicología, 28(2), 2012, 381 409.

Piaget, J., (1973). El nacimiento de la inteligencia en el niño. Ginebra: Neuschatêl, Delachaux et Niestlé. 
Rivera, A., (2016)- La atención durante el proceso de selección de la información en el aula. Journal of Kanda University of International Studies, 2016, (28):89-103.

Rugerio, J. y Guevara, Y., (2015). Alfabetización inicial y su desarrollo desde la educación infantil". Revisión del concepto e investigaciones aplicadas, (13), 2542. DOI:10.18239/ocnos_2015.13.02

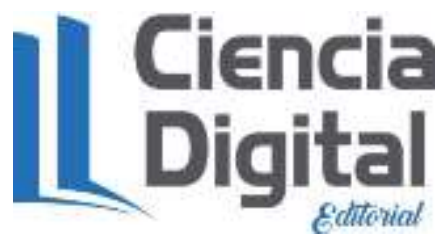




\section{PARA CITAR EL ARTÍCULO INDEXADO.}

Quimi Quimi, J. M., \& Maqueira Caraballo, G. de la C. (2021). La estimulación cognitiva. Base para el proceso de la lectoescritura en estudiantes con discapacidad intelectual. AlfaPublicaciones, 3(3.2), 69-80. https://doi.org/10.33262/ap.v3i3.2.100

\section{Ligital}

El artículo que se publica es de exclusiva responsabilidad de los autores y no necesariamente reflejan el pensamiento de la Revista Alfa Publicaciones.

El artículo queda en propiedad de la revista y, por tanto, su publicación parcial y/o total en otro medio tiene que ser autorizado por el director de la Revista Alfa Publicaciones.
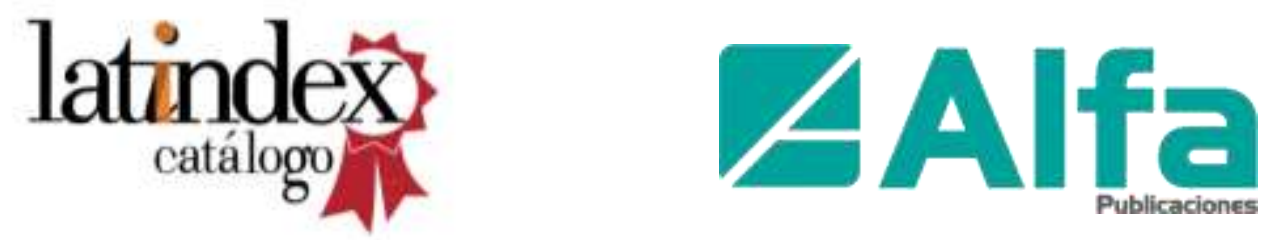

1 